\title{
MicroRNA-orchestrated pathophysiologic control in gut homeostasis and inflammation
}

\author{
Juneyoung Lee ${ }^{1,2}$, Eun Jeong Park ${ }^{1,3} \mathcal{E}$ Hiroshi Kiyono ${ }^{1,2,4, *}$ \\ ${ }^{1}$ Division of Mucosal Immunology, Department of Microbiology and Immunology, The Institute of Medical Science, The University of \\ Tokyo, Tokyo 108-8639, ${ }^{2}$ Department of Computational Biology and Medical Sciences, Graduate School of Frontier Sciences, The \\ University of Tokyo, Chiba 277-8561, ${ }^{3}$ Department of Molecular Pathobiology and Cell Adhesion Biology, Mie University Graduate School \\ of Medicine, Mie University, Mie 514-8507, ${ }^{4}$ International Research and Development Center for Mucosal Vaccines, The Institute of \\ Medical Science, The University of Tokyo, Tokyo 108-8639, Japan
}

The intestine represents the largest and most elaborate immune system organ, in which dynamic and reciprocal interplay among numerous immune and epithelial cells, commensal microbiota, and external antigens contributes to establishing both homeostatic and pathologic conditions. The mechanisms that sustain gut homeostasis are pivotal in maintaining gut health in the harsh environment of the gut lumen. Intestinal epithelial cells are critical players in creating the mucosal platform for interplay between host immune cells and luminal stress inducers. Thus, knowledge of the epithelial interface between immune cells and the luminal environment is a prerequisite for a better understanding of gut homeostasis and pathophysiologies such as inflammation. In this review, we explore the importance of the epithelium in limiting or promoting gut inflammation (e.g., inflammatory bowel disease). We also introduce recent findings on how small RNAs such as microRNAs orchestrate pathophysiologic gene regulation. [BMB Reports 2016; 49(5): 263-269]

\section{THE INTESTINE AS A HOTSPOT FOR HOMEOSTASIS AND INFLAMMATION}

The intestine is a unique organ where multiple communications between the immune system and commensal microbiota take place. It consists of diverse specialized cell types that function in distinct compartments, such as the epithelial layer, lamina propria, and gut-associated lymphoid tissue (GALT). Maintenance of intestinal homeostasis depends on elaborate interactions within and between these compartments; a break-

*Corresponding author. Tel: +81-3-5449-5270; Fax: +81-3-54495411; E-mail: kiyono@ims.u-tokyo.ac.jp

http://dx.doi.org/10.5483/BMBRep.2016.49.5.041

Received 26 February 2016

Keywords: Homeostasis, Inflammation, Intestine, MicroRNA, Pathophysiology down of host homeostasis can lead to inflammatory disorders such as inflammatory bowel disease (IBD). IBD, defined as chronic, uncontrolled mucosal inflammation within the gastrointestinal tract, is accompanied by diarrhea and abdominal pain (1). IBD is generally divided into two clinical phenotypes: Crohn's disease (CD), which affects the whole gastrointestinal tract (from the mouth to the anus), and ulcerative colitis (UC), which is limited mainly to the colon (1). The precise etiology of IBD is not completely understood, but it is clear that dynamic crosstalk between some genetic and environmental factors and the host immune system is critical for IBD pathogenesis (1). In this review, we introduce some key players (e.g., epithelial cells) in IBD pathophysiology and focus on the roles of microRNAs (miRNAs) in aggravating or ameliorating this inflammatory disorder.

\section{GENERAL FEATURES OF INTESTINAL EPITHELIAL CELLS}

The intestinal epithelium is composed of a single layer of intestinal epithelial cells (IECs) that covers the very large surface of the intestines (approximately $100 \mathrm{~m}^{2}$ ) and plays the foremost role as a physical barrier. IECs are important for the absorption of nutrients and they actively contribute to mucosal homeostasis by sensing antigens (e.g., commensal microbiota, food antigens, and pathogens) and eliciting immune responses. IECs include specialized cell types with distinct functions: intestinal stem cells (tissue self-renewal), transit amplifying cells (cell proliferation), Paneth cells (antimicrobial peptide secretion), enterocytes (nutrient absorption), goblet cells (mucus secretion), tuft cells (opioid release), enteroendocrine cells (endocrine signaling), and microfold $(M)$ cells (antigen sampling) $(2,3)$. One of the basic functions of IECs is to maintain intestinal homeostasis. IECs contribute to the barrier function by providing a first-line defense against continuous attack by invasive pathogens and heavy colonization by opportunistic bacteria. IECs also constitute a communication interface between bacteria in the lumen and leukocytes in intraepithelial and lamina propria compartments to mount local immune re-

ISSN: 1976-670X (electronic edition)

Copyright (c) 2016 by the The Korean Society for Biochemistry and Molecular Biology

(c) This is an open-access article distributed under the terms of the Creative Commons Attribution Non-Commercial License (http://creativecommons.org/licenses/by-nc/4.0) which permits unrestricted non-commercial use, distribution, and reproduction in any medium, provided the original work is properly cited. 
sponses $(2,3)$.

Turnover and shedding are important features of IECs (4). Their proliferation and differentiation from intestinal stem cells are regulated by key genes related to Wnt signaling (e.g., leucine rich repeat-containing G-protein coupled receptor 5 [Lgr5] and achaete-scute complex homolog 2 [ASCL2]) and the Notch pathway (e.g., Notch1 and Notch2) (5-7). The tight junction (T) proteins such as zonula occludens (ZO)-1 and integrins of aged IECs are altered to facilitate their detachment from the tip of the villus (small intestine) or crypt (colon), and removal (8). Under pathological conditions, the p53-PUMA (p53-upregulated modulator of apoptosis)/Noxa and immune cell-derived TNF- $\alpha /$ FasL pathways are thought to be involved in promoting IEC apoptosis (9-11), whereas receptor-interacting protein kinase 1 (RIPK1) is crucial for preventing IEC apoptosis (12). Thus, numerous intracellular signaling molecules maintain or alter gut homeostasis by regulating IEC apoptosis.

\section{ROLES OF IECs IN MAINTAINING GUT HOMEOSTASIS}

Antimicrobial peptides are essential components of the host immune system. IECs constitutively produce antimicrobial peptides to regulate microbial colonization and penetration of intestinal epithelium $(13,14)$. The primary function of Paneth cells, which are long-lived cells located at the base of the crypts of Lieberkühn in the small intestine, is to secrete (in an inducible manner) various antimicrobial peptides and proteins such as lysozyme, $\alpha$-defensins (e.g., human $\alpha$-defensins 5 [HD5] and 6 [HD6] and cryptdins in mice), C-type lectins of the Reg III (regenerating islet-derived protein 3) family, cathelicidins, and lipocalins (15). They play important roles in the susceptibility to IBD; this susceptibility is conferred by mutations in NOD2 (nucleotide-binding oligomerization domaincontaining protein 2) $(16,17)$. For instance, reduced expression of defensins in Paneth cells, which may impair barrier function, is one of the characteristics of IBD, especially ileal $C D$ (16). Insufficient amounts of Wnt ligands in ileal CD patients' monocytes, but not in lymphocytes, are associated with decreased defensin formation (17). ATG16L1 (autophagy-related 16-like 1) has been suggested to control granule exocytosis (18) and XBP1 (X-box binding protein 1) has been suggested to maintain Paneth cell function (19). Other defensins such as human neutrophil peptides (HNPs) are secreted from neutrophil granules $(20,21)$. Beta-defensins (e.g., hBD-1) are expressed mainly in goblet cells, enterocytes, and Paneth cells (22). LL37/hCAP-18 (human cathelicidin LL-37/human cationic antimicrobial peptide 18) expressed in mature enterocytes plays a principal role in protecting the intestinal mucosal surface (23). Various antimicrobial peptides produced by intestinal epithelium are thus considered to be important for homeostasis in the digestive tract.

\section{INFLAMMATORY MEDIATORS IN IBD}

Genetic, immunologic, and environmental factors are implicated in IBD. Genome-wide association studies (GWAS) have demonstrated the genetic architecture of pathways related to IBD; for example, some of the IBD-related loci that have been identified include IBD1 (NOD2) and IBD3 (HLA-region) (24-26). However, enormous heterogeneity in genetic or epigenetic etiology observed among IBD patients has indicated the importance of experimental models that recapitulate pathologic and symptomatic features of human IBD. In this section, we introduce a few key players that mediate pathophysiologic exacerbation of IBD.

\section{TNF- $\alpha$}

TNF- $\alpha$ is produced mainly by leukocytes (e.g., lymphocytes, activated monocytes, and macrophages) (27) and is a crucial pro-inflammatory cytokine. Binding of TNF- $\alpha$ to its receptors (TNFRs) elevates the levels of pro-inflammatory molecules such as adhesion molecules (e.g., intercellular adhesion molecule-1, vascular cell adhesion molecule-1, and mucosal addressin cell adhesion molecule-1), cytokines (e.g., IL-1 $\beta$ and IL-6), and chemokines (e.g., CCL19, CCL21, CXCL12, and CXCL13) (28). TNF- $\alpha$ also reduces the barrier function of IECs in IBD by inducing TNFR1-mediated IEC apoptosis (29) and increasing the permeability of TJ through TNFR2 signaling (30). TNF- $\alpha$ modulates immunological self-tolerance in IBD, because it inhibits the suppressive function of regulatory $T$ cells $\left(\mathrm{T}_{\text {reg }}\right)$ by activating the NF- $\kappa B$ pathway (31). Consequently, many anti-TNF- $\alpha$ therapies have been suggested for the treatment of IBD, including infliximab (IFX), adalimumab (ADA), golimumab (GLM), and certolizumab pegol (CZP) $(32,33)$. However, these treatments have some adverse effects (including malignancy); therefore, novel or modified therapies with increased selectivity are very much needed (34).

\section{IL-6}

IL-6 is a central cytokine in IBD pathology. A remarkable increase in IL-6 in the sera (35) and inflamed colonic mucosa (36) of IBD patients has been reported. The ameliorating effect of anti-IL-6 receptor mAb administration has been reported in a model of CD45RB high $\mathrm{CD} 4+\mathrm{T}$ cell-induced colitis in SCID mice (37). Blockade of IL-6-mediated activation reduced the expression of the inflammatory cytokines, IFN- $\gamma$, TNF- $\alpha$, and IL-1 $\beta$, suggesting its therapeutic potential for the treatment of $\mathrm{CD}(37)$.

\section{STAT3}

The transcription factor, signal transducer and activator of transcription 3 (STAT3), acts downstream of IL-6 (38, 39). IL-6-induced T-cell proliferation is significantly reduced by STAT3 deficiency; in the presence of IL-6, STAT3-deficient T cells undergo apoptosis (40). Hyper-activation of STAT3 occasionally results in epithelial cell apoptosis (41). Among several mem- 
bers of the STAT family (e.g., STAT1, STAT3, STAT5, and STAT6), phosphorylation of STAT3 is prominent in the colon tissue of IBD patients and in mice with dextran sulfate sodium (DSS)-induced colitis (42). STAT3 phosphorylation was also observed in chronic models of T cell-dependent colitis (42). Accordingly, STAT3 is referred to as a 'double-edged sword' in the development of inflammation.

\section{CHARACTERISTICS OF SMALL RNA BIOGENESIS AND FUNCTION}

In this section, we focus on recent findings on the role of small RNAs, especially miRNAs, in intestinal inflammation. Small RNAs of 20-30 nucleotides (nt) are grouped into three major categories: endogenous small interfering RNA (endo-siRNA), Piwi-interacting RNA (piRNA), and miRNA (43). They are present in eukaryotes from yeast to humans and they regulate homeostasis by targeting chromatin and transcripts $(44,45)$. endo-siRNA are double-strand RNA-derived small RNAs that share some features with miRNAs, including the use of Dicer and Argonaute proteins in their processing (44). piRNAs are RNase III-independent small RNAs that function in transposon silencing; they are processed by Zucchini and PIWI proteins (45). miRNAs are predominant small RNAs in most tissues of multicellular organisms (46).

miRNAs are small, endogenous, non-coding, single-stranded RNAs that modulate gene expression during cell development, differentiation, and apoptosis (47). In comparison with other types of small RNAs, their biogenesis pathways and functional roles have been extensively investigated (43). Mature miRNAs post-transcriptionally regulate protein-coding genes by base pairing between their 5 '-end seed regions (nucleotide positions 2-7) and the 3'-untranslated regions (UTR) of target mRNAs, which leads to translational repression or mRNA degradation (48). The canonical multi-step miRNA biogenesis is performed mainly by two processing enzymes, the nuclear RNase III Drosha (49) and the cytoplasmic RNase III Dicer (50). The involvement of miRNAs in IBD - not only their functional targets and mechanisms, but also their biomarker discovery and therapeutic use- has been extensively investigated.

\section{miRNAs AS TRIGGERS OF IBD PROGRESSION}

Diverse factors affect the development of IBD (see above), but its exact etiology and pathophysiology remain elusive. However, a few microRNAs have shown consistent results in multiple studies and may be useful as IBD biomarkers. Here, we introduce the well-known miRNAs (miR-21, miR-155, and miR-223) and novel miRNAs (miR-1224-5p, miR-3473a, and miR-5128) that were predicted to have colitogenic ability in the gut epithelium (Table 1).

\section{miR-21}

miR-21 is one of the most studied miRNAs that are implicated in many inflammatory diseases, including IBD. miR-21 is significantly elevated in the blood of patients with UC, together with other signature miRNAs (e.g., miR-16, miR-28-5p, miR-151-5p, miR-155, and miR-199a-5p) (48). However, miR-21 is not increased in the blood of CD patients (51). Given that UC and CD are phenotypically distinct, this circulating miRNA turned out to be useful in distinguishing these two major phenotypes of IBD at diagnosis. The importance of miR-21 in UC has been emphasized in other reports (52-54). Microarray analysis and quantitative PCR showed miR-21 upregulation in the inflamed colon tissue of patients with active UC (52). Another study focused on the role of miR-21 in epithelial barrier function and reported that miR-21 accumulated in the intestinal epithelium of UC patients (53). This study also showed that overexpression of miR-21 reduced trans-epithelial electrical resistance and increased inulin permeability by disrupting intestinal epithelial TJ. Finally, RhoB (Ras homolog gene family, member B) was suggested as a putative target of miR-21 (53). The unique role of miR-21 was recently described in a murine colitis model: miR-21 knockout protected mice from acute experimental colitis (54). miR-21 was found to regulate the expression of RhoB and Cdc42 (cell division cycle 42), which are associated with intestinal epithelial function (54). Taken together, the available data indicate that miR-21 plays a pivotal role in regulation of gene expression in IBD.

Table 1. miRNAs and their putative targets implicated in inflammatory bowel disease

\begin{tabular}{|c|c|c|c|c|}
\hline miRNAs & Expression & Tissue or cell type & Targets & References \\
\hline miR-21 & Increased & Blood and colon in $\mathrm{UC}^{\mathrm{a}}$ & RhoB, Cdc42 & $(51-4)$ \\
\hline miR-155 & Increased & Colon in UC & Foxp3, SOCS1 & $(55-0)$ \\
\hline miR-223 & Decreased & Myeloid cells & C/EBP $\beta$ & $(61-4)$ \\
\hline miR-1224-5p & Increased & Inflamed large-IECs ${ }^{\mathrm{b}}$ & AQP8 & $(65)$ \\
\hline miR-3473a & Increased & Inflamed large-IECs & AQP8 & (65) \\
\hline miR-5128 & Increased & Inflamed large-IECs & ABCG2, AQP8 & (65) \\
\hline
\end{tabular}

${ }^{a} U C$, ulcerative colitis. ${ }^{b}$ Large-IECs, epithelial cells of the large intestine. 


\section{miR-155}

miR-155 is important in hematopoietic-derived immune cells (55-57). Both the frequency and the absolute number of $T_{\text {reg }}$ cells are diminished in the lymphoid organs (such as spleen, thymus, and lymph nodes) of miR- $155^{-l-}$ mice, although the intrinsic suppressive function of $\mathrm{T}_{\text {reg }}$ cells is not impaired (55). As Foxp3 is a target of miR-155 (56), the miR-155/Foxp3 axis may be important in transcriptional programming for $\mathrm{T}_{\text {reg }}$ cell development (55). In line with the role of miR-155 in T cells, miR-155 deficiency ameliorates the pathogenesis of experimental colitis by downregulating the Th1/Th17 response (57). miR-155 also has a modulatory function in mesenchymal cells from colon tissues of patients with UC: miR-155 upregulation in intestinal myofibroblasts decreases the level of SOCS1 (suppressor of cytokine signaling 1); and SOCS1 is a direct target of miR-155 (58). An important observation is that both miR-21 and miR-155 are critical for controlling IBD: they promote microsatellite instability (MSI) (59), which results from impaired DNA mismatch repair (MMR), and may induce $\mathrm{K}$-ras mutations in colonic mucosa and thus aggravate UC (60). These two miRNAs act as a link between IBD and colorectal cancer by targeting MMR (59). Therefore, miR-21 and miR-155 can be considered as targets for IBD treatment.

\section{miR-223}

Dendritic cells (DCs) and macrophages are important for orchestrating both host immune tolerance and protective immune responses (61). Recently, a novel function of miR-223 was identified in DCs and macrophages of the small intestine (62). miR-223 is highly expressed in myeloid cells and regulates granulocyte production and function $(63,64)$. Depletion of miR-223 significantly reduces the number of CX3CR $1^{\text {high }}$ macrophages at steady state and enhances the pro-inflammatory phenotype of macrophages, $\mathrm{CD}_{103}{ }^{+}$conventional DCs (cDCs), and monocyte-derived DCs (moDCs). miR-223 KO mice had more severe colitis than wild-type (62). miR-223 expressed in myeloid cells is thought to be an essential regulator of intestinal homeostasis.

The level of miR-223-3p is increased in inflamed epithelial cells of the small intestine (small-IECs) and large intestine (large-IECs) of mice with DSS-induced colitis (65). Intriguingly, miRNA profiling and network analysis showed that miR-223-3p regulates its target genes differentially in small- and large-IECs (Fig. 1) (65). Thus, cell-based bioinformatics proved to be a powerful tool for the identification of etiologic or regulatory factors that determine distinct pathophysiology of small and large intestines.

\section{miR-1224-5p, miR-3473a, and miR-5128}

miRNAs are considered to be a reliable genetic source for understanding the pathogenesis of IBD. Most studies have focused on the markedly altered expression of miRNAs and their target genes in tissues or cell lines $(66,67)$. Live IECs sampled from IBD models could be useful for revealing the basis for pathophysiological differences between different regions of intestines (65). Furthermore, establishing putative networks of gene expression regulation by miRNAs in either normal or inflamed IECs helps to comprehensively understand IBD pathophysiology, especially in relation to the barrier function and communication. In a mouse colitis model, a more complex network in large-IECs than in small-IECs could demonstrate a correlation between $\mathrm{UC}$ severity and the complexity of miRNA networks. Among putative biomarkers in inflamed large-IECs, miRNAs (e.g., miR-1224-5p, miR-3473a, or miR-5128) and their targets ABCG2 (ATP-binding cassette transporter G2) or AQP8 (aquaporin 8) appear to be involved in IBD development and progression (65). These biomarkers remain to be

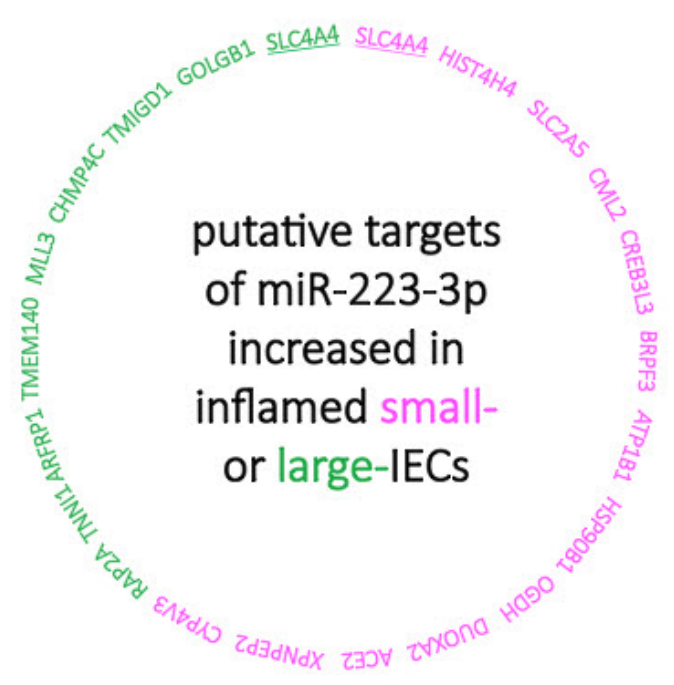

Fig. 1. Networks between miR-223-3p and its putative target genes distinctive to inflamed small- (pink) or large-IECs (green). miR223-3p was up-regulated in both small- and large-IECs of the mice with DSS-induced colitis (65). Microarray and TargetScan database analyses suggest that miR-223-3p exclusively down-regulates different target genes between both inflamed intestines except for SLC4A4 (solute carrier family 4 anion exchanger) (underlines): HIST4H4 (histone cluster 4, H4), SLC2A5 (solute carrier family 2 member 5), CML2 (camello-like 2), CREB3L3 (cAMP responsive element binding protein 3-like 3), BRPF3 (bromodomain and PHD finger containing 3), ATP1B1 (ATPase, $\mathrm{Na}^{+} / \mathrm{K}^{+}$transporting, beta 1 polypeptide), HSP90B1 (heat shock protein 90, beta, member 1), $\mathrm{OGDH}$ (oxoglutarate dehydrogenase), DUOXA2 (dual oxidase maturation factor 2), ACE2 (angiotensin I converting enzyme 2), XPNPEP2 (X-prolyl aminopeptidase 2), and CYP4V3 (cytochrome P450, family 4, subfamily $\mathrm{v}$, polypeptide 3 ) were markedly reduced in inflamed, compared with normal, small-IECs; GOLGB1 (golgin subfamily b macrogolgin 1), TMIGD1 (transmembrane and immunoglobulin domain containing 1), CHMP4C (chromatin modifying protein 4C), MLL3 (myeloid/lymphoid or mixed-lineage leukemia 3), TMEM140 (transmembrane protein 140), ARFRP1 (ADP-ribosylation factor related protein 1), TNNI1 (troponin I, skeletal, slow 1), and RAP2A (RAS related protein 2a) were significantly down-regulated in inflamed, compared with normal, large-IECs. 
functionally characterized in vivo and in vitro to further clarify their contributions to IBD pathology. Taken together, current elaborate studies will provide clues for understanding the pathophysiologic mechanisms in the gut and for the discovery of miRNA biomarkers and their specific gene regulatory networks in IBD.

\section{CONCLUSIONS AND PERSPECTIVES}

Considerable progress has been made in our understanding of IBD pathophysiology. It is currently accepted that one of the main causes of IBD is imbalanced immunological communication between enteric microbiota and effector immune cells across the epithelial interface. Research on miRNAs has focused on alteration of their expression at the tissue level in human IBD patients or in animal models and elucidation of the role of target gene silencing in disruption of gut homeostasis to trigger the development of IBD. miRNAs and their target genes can be considered as promising targets for IBD therapeutics. Despite the potential applicability of miRNAs or their related molecules in treating IBD, substantial limitations remain, including insufficiency of comprehensive profiles of miRNA expression or established networks specific to relevant cell types; variability of miRNA expression among patients or within tissues; and lack of adequate methods for in vivo RNAi delivery into target cells or tissues. Validation of miRNA functions in vivo and in vitro will help to acquire further valid scientific knowledge of IBD pathophysiology that is needed for diagnostics and therapy.

\section{ACKNOWLEDGEMENTS}

This work was supported by grants from the Core Research for Evolutional Science and Technology Program of the Japan Science and Technology Agency (H.K.); the Ministry of Education, Culture, Sports, Science, and Technology of Japan (Grant-in-Aid for Scientific Research S) (H.K.); and the IMSUT Joint Research Project at the Institute of Medical Science in the University of Tokyo (E.J.P. and H.K.). We thank all members of our laboratories for their discussion and technical support.

\section{REFERENCES}

1. Kaser A, Zeissig S and Blumberg RS (2010) Inflammatory bowel disease. Annu Rev Immunol 28, 573-621

2. Artis D (2008) Epithelial-cell recognition of commensal bacteria and maintenance of immune homeostasis in the gut. Nat Rev Immunol 8, 411-420

3. Hooper LV and Macpherson AJ (2010) Immune adaptations that maintain homeostasis with the intestinal microbiota. Nat Rev Immunol 10, 159-169

4. Creamer B, Shorter RG and Bamforth J (1961) The turnover and shedding of epithelial cells. I. The turnover in the gastro-intestinal tract. Gut 2, 110-118

5. Barker N, van Es JH, Kuipers J et al (2007) Identification of stem cells in small intestine and colon by marker gene Lgr5. Nature 449, 1003-1007

6. van der Flier LG, van Gijn ME, Hatzis P et al (2009) Transcription factor achaete scute-like 2 controls intestinal stem cell fate. Cell 136, 903-912

7. Riccio O, van Gijn ME, Bezdek AC et al (2008) Loss of intestinal crypt progenitor cells owing to inactivation of both Notch1 and Notch2 is accompanied by derepression of CDK inhibitors p27Kip1 and p57Kip2. EMBO Rep 9, 377-383

8. Guan Y, Watson AJ, Marchiando AM et al (2011) Redistribution of the tight junction protein ZO-1 during physiological shedding of mouse intestinal epithelial cells. Am J Physiol Cell Physiol 300, C1404-1414

9. Nakano K and Vousden KH (2001) PUMA, a novel proapoptotic gene, is induced by p53. Mol Cell 7, 683-694

10. Oda E, Ohki R, Murasawa $H$ et al (2000) Noxa, a BH3-only member of the Bcl-2 family and candidate mediator of p53-induced apoptosis. Science 288, 1053-1058

11. Chopra M, Brandl A, Siegmund D et al (2015) Blocking TWEAK-Fn14 interaction inhibits hematopoietic stem cell transplantation-induced intestinal cell death and reduces GVHD. Blood 126, 437-444

12. Takahashi N, Vereecke L, Bertrand MJ et al (2014) RIPK1 ensures intestinal homeostasis by protecting the epithelium against apoptosis. Nature 513, 95-99

13. Salzman NH, Hung K, Haribhai D et al (2010) Enteric defensins are essential regulators of intestinal microbial ecology. Nat Immunol 11, 76-83

14. Cash HL, Whitham CV, Behrendt CL and Hooper LV (2006) Symbiotic bacteria direct expression of an intestinal bactericidal lectin. Science 313, 1126-1130

15. Koslowski MJ, Beisner J, Stange EF and Wehkamp J (2010) Innate antimicrobial host defense in small intestinal Crohn's disease. Int J Med Microbiol 300, 34-40

16. Wehkamp J, Salzman NH, Porter E et al (2005) Reduced Paneth cell alpha-defensins in ileal Crohn's disease. Proc Natl Acad Sci U S A 102, 18129-18134

17. Courth LF, Ostaff MJ, Mailander-Sanchez D, Malek NP, Stange EF and Wehkamp J (2015) Crohn's disease-derived monocytes fail to induce Paneth cell defensins. Proc Natl Acad Sci U S A 112, 14000-14005

18. Cadwell K, Liu JY, Brown SL et al (2008) A key role for autophagy and the autophagy gene Atg16l1 in mouse and human intestinal Paneth cells. Nature 456, 259-263

19. Kaser A, Lee AH, Franke A et al (2008) XBP1 links ER stress to intestinal inflammation and confers genetic risk for human inflammatory bowel disease. Cell 134, 743756

20. Ganz T, Selsted ME, Szklarek D et al (1985) Defensins. Natural peptide antibiotics of human neutrophils. I Clin Invest 76, 1427-1435

21. Wilde CG, Griffith JE, Marra MN, Snable JL and Scott RW (1989) Purification and characterization of human neutrophil peptide 4, a novel member of the defensin family. J Biol Chem 264, 11200-11203

22. O'Neil DA, Porter EM, Elewaut D et al (1999) Expression and regulation of the human beta-defensins hBD-1 and hBD-2 in intestinal epithelium. J Immunol 163, 67186724 
23. Hase K, Eckmann L, Leopard JD, Varki N and Kagnoff MF (2002) Cell differentiation is a key determinant of cathelicidin LL-37/human cationic antimicrobial protein 18 expression by human colon epithelium. Infect Immun 70, 953-963

24. Barrett JC, Hansoul S, Nicolae DL et al (2008) Genomewide association defines more than 30 distinct susceptibility loci for Crohn's disease. Nat Genet 40, 955-962

25. Van Limbergen J, Wilson DC and Satsangi J (2009) The genetics of Crohn's disease. Annu Rev Genomics Hum Genet 10, 89-116

26. Imielinski M, Baldassano RN, Griffiths A et al (2009) Common variants at five new loci associated with early-onset inflammatory bowel disease. Nat Genet 41, 1335-1340

27. Peake ST, Bernardo D, Mann ER, AI-Hassi HO, Knight SC and Hart AL (2013) Mechanisms of action of anti-tumor necrosis factor alpha agents in Crohn's disease. Inflamm Bowel Dis 19, 1546-1555

28. Tracey D, Klareskog L, Sasso EH, Salfeld JG and Tak PP (2008) Tumor necrosis factor antagonist mechanisms of action: a comprehensive review. Pharmacol Ther 117, 244-279

29. Inagaki-Ohara K, Yada S, Takamura N et al (2001) p53-dependent radiation-induced crypt intestinal epithelial cells apoptosis is mediated in part through TNF-TNFR1 system. Oncogene 20, 812-818

30. Liu H, Li M, Wang P and Wang F (2011) Blockade of hypoxia-inducible factor-1alpha by YC-1 attenuates interferon-gamma and tumor necrosis factor-alpha-induced intestinal epithelial barrier dysfunction. Cytokine 56, 581588

31. Nagar M, Jacob-Hirsch J, Vernitsky H et al (2010) TNF activates a NF-kappaB-regulated cellular program in human CD45RA- regulatory $\mathrm{T}$ cells that modulates their suppressive function. J Immunol 184, 3570-3581

32. Biancheri P, Brezski RJ, Di Sabatino A et al (2015) Proteolytic cleavage and loss of function of biologic agents that neutralize tumor necrosis factor in the mucosa of patients with inflammatory bowel disease. Gastroenterology 149, 1564-1574 e1563

33. Nielsen $\mathrm{OH}$ and Ainsworth MA (2013) Tumor necrosis factor inhibitors for inflammatory bowel disease. N Engl J Med 369, 754-762

34. Melmed GY and Targan SR (2010) Future biologic targets for IBD: potentials and pitfalls. Nat Rev Gastroenterol Hepatol 7, 110-117

35. Mahida YR, Kurlac L, Gallagher A and Hawkey CJ (1991) High circulating concentrations of interleukin- 6 in active Crohn's disease but not ulcerative colitis. Gut 32, 15311534

36. Mitsuyama K, Sasaki E, Toyonaga A et al (1991) Colonic mucosal interleukin-6 in inflammatory bowel disease. Digestion 50, 104-111

37. Yamamoto M, Yoshizaki K, Kishimoto T and Ito $\mathrm{H}$ (2000) IL-6 is required for the development of Th1 cell-mediated murine colitis. J Immunol 164, 4878-4882

38. Akira S, Nishio Y, Inoue M et al (1994) Molecular cloning of APRF, a novel IFN-stimulated gene factor 3 p91-related transcription factor involved in the gp130-mediated sig- naling pathway. Cell 77, 63-71

39. Zhong Z, Wen Z and Darnell JE Jr (1994) Stat3: a STAT family member activated by tyrosine phosphorylation in response to epidermal growth factor and interleukin-6. Science 264, 95-98

40. Takeda K, Kaisho T, Yoshida N, Takeda J, Kishimoto T and Akira S (1998) Stat3 activation is responsible for IL-6-dependent $\mathrm{T}$ cell proliferation through preventing apoptosis: generation and characterization of T cell-specific Stat3-deficient mice. J Immunol 161, 4652-4660

41. Suzuki A, Hanada T, Mitsuyama K et al (2001) CIS3/ SOCS3/SSI3 plays a negative regulatory role in STAT3 activation and intestinal inflammation. J Exp Med 193, 471481

42. Chapman RS, Lourenco PC, Tonner E et al (1999) Suppression of epithelial apoptosis and delayed mammary gland involution in mice with a conditional knockout of Stat3. Genes Dev 13, 2604-2616

43. Kim VN, Han J and Siomi MC (2009) Biogenesis of small RNAs in animals. Nat Rev Mol Cell Biol 10, 126-139

44. Ghildiyal M and Zamore PD (2009) Small silencing RNAs: an expanding universe. Nat Rev Genet 10, 94-108

45. Ishizu H, Siomi H and Siomi MC (2012) Biology of PIWl-interacting RNAs: new insights into biogenesis and function inside and outside of germlines. Genes Dev 26, 2361-2373

46. Ha M and Kim VN (2014) Regulation of microRNA biogenesis. Nat Rev Mol Cell Biol 15, 509-524

47. Bushati $N$ and Cohen SM (2007) microRNA functions. Annu Rev Cell Dev Biol 23, 175-205

48. Saunders MA, Liang $\mathrm{H}$ and Li WH (2007) Human polymorphism at microRNAs and microRNA target sites. Proc Natl Acad Sci U S A 104, 3300-3305

49. Lee Y, Ahn C, Han J et al (2003) The nuclear RNase III Drosha initiates microRNA processing. Nature 425, 415-419

50. Chendrimada TP, Gregory RI, Kumaraswamy $\mathrm{E}$ et al (2005) TRBP recruits the Dicer complex to Ago2 for microRNA processing and gene silencing. Nature 436, 740-744

51. Paraskevi A, Theodoropoulos G, Papaconstantinou I, Mantzaris G, Nikiteas N and Gazouli M (2012) Circulating MicroRNA in inflammatory bowel disease. J Crohns Colitis 6, 900-904

52. Takagi T, Naito Y, Mizushima K et al (2010) Increased expression of microRNA in the inflamed colonic mucosa of patients with active ulcerative colitis. J Gastroenterol Hepatol 25 Suppl 1, S129-133

53. Yang Y, Ma Y, Shi C et al (2013) Overexpression of miR-21 in patients with ulcerative colitis impairs intestinal epithelial barrier function through targeting the Rho GTPase RhoB. Biochem Biophys Res Commun 434, 746-752

54. Shi C, Liang Y, Yang J et al (2013) MicroRNA-21 knockout improve the survival rate in DSS induced fatal colitis through protecting against inflammation and tissue injury. PLoS One 8, e66814

55. Kohlhaas S, Garden OA, Scudamore C, Turner $M$, Okkenhaug $\mathrm{K}$ and Vigorito $\mathrm{E}$ (2009) Cutting edge: the Foxp3 target miR-155 contributes to the development of 
regulatory T cells. J Immunol 182, 2578-2582

56. Zheng Y, Josefowicz SZ, Kas A, Chu TT, Gavin MA and Rudensky AY (2007) Genome-wide analysis of Foxp3 target genes in developing and mature regulatory $\mathrm{T}$ cells. Nature 445, 936-940

57. Singh UP, Murphy AE, Enos RT et al (2014) miR-155 deficiency protects mice from experimental colitis by reducing $\mathrm{T}$ helper type 1/type 17 responses. Immunology 143, 478-489

58. Pathak S, Grillo AR, Scarpa M et al (2015) MiR-155 modulates the inflammatory phenotype of intestinal myofibroblasts by targeting SOCS1 in ulcerative colitis. Exp Mol Med 47, e164

59. Svrcek M, El-Murr N, Wanherdrick K et al (2013) Overexpression of microRNAs- 155 and 21 targeting mismatch repair proteins in inflammatory bowel diseases. Carcinogenesis 34, 828-834

60. Lyda MH, Noffsinger A, Belli J and Fenoglio-Preiser CM (2000) Microsatellite instability and K-ras mutations in patients with ulcerative colitis. Hum Pathol 31, 665-671

61. Coombes JL and Powrie F (2008) Dendritic cells in intestinal immune regulation. Nat Rev Immunol 8, 435-446
62. Zhou H, Xiao J, Wu N et al (2015) MicroRNA-223 regulates the differentiation and function of intestinal dendritic cells and macrophages by targeting C/EBPbeta. Cell Rep $13,1149-1160$

63. Chen CZ, Li L, Lodish HF and Bartel DP (2004) MicroRNAs modulate hematopoietic lineage differentiation. Science 303, 83-86

64. Johnnidis JB, Harris MH, Wheeler RT et al (2008) Regulation of progenitor cell proliferation and granulocyte function by microRNA-223. Nature 451, 1125-1129

65. Lee J, Park EJ, Yuki Y et al (2015) Profiles of microRNA networks in intestinal epithelial cells in a mouse model of colitis. Sci Rep 5, 18174

66. Nguyen HT, Dalmasso G, Muller S, Carriere J, Seibold F and Darfeuille-Michaud A (2014) Crohn's disease-associated adherent invasive Escherichia coli modulate levels of microRNAs in intestinal epithelial cells to reduce autophagy. Gastroenterology 146, 508-519

67. Archanioti P, Gazouli M, Theodoropoulos G, Vaiopoulou A and Nikiteas N (2011) Micro-RNAs as regulators and possible diagnostic bio-markers in inflammatory bowel disease. J Crohns Colitis 5, 520-524 